\title{
XiangshaLiujunzi decoction alleviates the symptoms of functional dyspepsia by regulating brain-gut axis and production of neuropeptides

Jing Liu ${ }^{1 \dagger}$, Feng $\mathrm{Li}^{1^{*}}$, Xu-Dong Tang ${ }^{2 \dagger}$, Jie $\mathrm{Ma}^{1+}$, Xin Ma', Dong-Yu Ge${ }^{1}$, Gen-Mao Li ${ }^{1}$ and Yong Wang ${ }^{1}$

\begin{abstract}
Background: Chinese medicine xiangshaliujunzi decoction (XSLZD) plays a key role in treating functional dyspepsia (FD), a common clinical gastrointestinal disorder. However, the mechanism of this disease is unclear. Brain-gut axis regulates food intake behaviour, and this regulatory mechanism is mediated by neuropeptides. Brain-gut axis impairment and neuropeptide alteration may be the pathological mechanisms of FD, and brain-gut axis regulation may influence the action of medicine.
\end{abstract}

Methods: In our experiment, the effect of XSLZD on FD was evaluated in terms of food intake, sucrose preference test and electromyogram. Changes in neuropeptides [ghrelin, cholecystokinin (CCK) and vasoactive intestinal polypeptide (VIP)] were detected through immunohistochemistry, real-time PCR and ELISA.

Results: XSLJZD increased food intake and the percentage of sucrose preference (>75\%). However, the response to gastric detention decreased. Furthermore, XSLZD increased ghrelin, CCK, VIP proteins and genes in the stomach. XSLJZD also increased ghrelin, CCK and VIP proteins in serum. By contrast, XSLJZD decreased the mRNA expression of these neuropeptides in the hypothalamus.

Conclusions: XSLJZD alleviated the symptoms of FD by upregulating the production of ghrelin, CCK and VIP and by increasing the levels of these neuropeptides in circulation. This finding can help elucidate the mechanism of FD and can provide further insight into the pharmacokinetics of XSLJZD.

Keywords: Xiangshaliujunzi decoction, Functional dyspepsia, Brain-gut axis, Brain-gut peptide

\section{Background}

Functional dyspepsia (FD) is a common clinical gastrointestinal disorder characterised by persistent or recurrent pain and discomfort. This discomfort is mainly experienced in the upper abdomen without evidence of organic structural abnormalities associated with these symptoms. In a household survey, approximately $25 \%$ of the normal population in the United States suffers from FD [1]. In China, a definite statistics for FD is lacking; however, several works indicate that 8.66 to $11 \%$ of patients opt for hospital admission because of abdominal fullness [2-4]. A number of pathophysiological mechanisms have been proposed to explain several clinical

\footnotetext{
*Correspondence: lifeng95@vip.sina.com

${ }^{\dagger}$ Equal contributors

'School of Preclinical Medicine, Beijing University of Chinese Medicine, Beijing, China

Full list of author information is available at the end of the article
}

symptoms, including hypersensitivity to gastric distention, impaired gastric accommodation of a meal, delayed gastric emptying, altered duodenal sensitivity to lipids or acid, abnormal duodenojejunal motility and central nervous system (CNS) dysfunction; however, the evidence related to these symptoms varies in patients $[5,6]$. Hence, the definite mechanism remains unknown.

FD, an example of functional gastrointestinal disorders, is a common pathological condition affecting the gut, which is controlled by the nervous system [7]. The gastrointestinal tract (GIT) and the nervous system, including CNS and enteric nervous system (ENS), are involved in a two-way extrinsic communication by parasympathetic and sympathetic nerves. These nerves contain efferent fibres and afferent sensory fibres required for gut-brain signalling. Afferent nerves comprise many sensors at the terminals in the gut related to visceral mechano-, chemo-, and 
noci-receptors; when excited, these sensors may trigger various visceral reflexes that regulate GIT functions, including appetite [8-10]. The disorders affecting the regulation of the two-way communication between the brain and the gut (brain-gut axis) are important in the pathogenesis of these diseases [11]. Neuropeptides are important mediators in the nervous system and between neurons and other cell types. Neuropeptides, such as ghrelin, cholecystokinin (CCK) and vasoactive intestinal polypeptide (VIP), are possibly implicated in the bidirectional gut-brain communication [12]. Ghrelin is a 28-amino acid peptide hormone [13], which is produced predominantly by P/D1 cells of the gastric oxyntic gland; this hormone is mainly found in the proximal stomach $[14,15]$. Ghrelin is involved in many biological activities because this hormone plays autocrine and paracrine roles in regulatory processes, such as regulation of appetite, gut motility, growth hormone release, immunomodulation [16, 17] and initiation of food intake under neural control [18]. CCK belongs to the gut-brain family of peptide hormones [19]. This hormone is secreted by the gastrointestinal system in response to food intake; furthermore, CCK is released by specialised neurons in the myenteric plexus and the brain [20]. In a previous study, intravenous injection of CCK suppresses hunger and feeding in humans [21-23]. CCK also participates in signal transduction in the braingut axis via the primary afferent fibres of the vagus nerve, and the same fibres probably trigger the expression of receptors for ghrelin [24]. VIP, a 28-amino acid neuropeptide, is distributed in central and peripheral neurons; this neuropeptide is involved in many physiological intestinal functions, such as motility regulation, secretory activity and vasodilatation, peristaltic reflex inhibition in the circular smooth muscle layer and sphincter relaxation [25]. Neuronal VIP is also a mediator of neural response to aspirin-induced stomach inflammatory state [26]. These studies demonstrated that the disorder of the brain-gut axis may be the pathogenesis of FD.

Xiangshaliujunzi decoction (XSLJZD), a classic decoction used during Qing Dynasty in China, plays a key role in the treatment of FD; XSLJZD is more effective than prokinetic drugs in the treatment of this disease [27]. However, the mechanism by which XSLJZD relieves FD remains unknown. We studied the mechanism of the modified XSLJZD on the perspective of the brain-gut axis and neuropeptides. This regulatory mechanism can be the specific mode to treat FD.

\section{Methods}

\section{Animals}

Male Sprague-Dawley rats were used in all of the experiments (SPF Laboratory Animal Technology Co., Ltd., Beijing, China). The experiments were performed in accordance with the Guide for the Care and Use of
Laboratory Animals published by the National Institutes of Health (NIH Publications No. 85-23, revised 1996) and with the approval of the Animal Care Committee of Beijing Medical Centre. The 10-day-old rat pups received $0.2 \mathrm{~mL}$ of $0.1 \%$ iodoacetamide (IA) in $2 \%$ sucrose by oral gavage daily for 6 days. The control group received $0.2 \mathrm{~mL}$ of $2 \%$ sucrose [28]. The 6-week-old IA-treated rats were randomly divided into four groups: model group $(n=12$; received same volume of water as vehicle), XSLJZD-treated group ( $n=12$; treated with XSLJZD), low-dose XSLJZDtreated group ( $n=12$; treated with half dose of XSLJZD) and domperidone-treated group $(n=12)$. The 6-week-old sucrose-treated rats were designated as the control group $(n=12)$. The 6 -week-old rats received $5 \mathrm{~mL} / \mathrm{kg}$ of each drug or water daily by oral gavage for 10 days.

\section{Drugs}

XSLJZD is composed of eight different Chinese medicinal herbs (Table 1). The components were prepared by the Pharmaceutical Department of Xiyuan Hospital, affiliated with Chinese Academy of TCM. Pure extracts of the components were prepared. The components were dissolved in water. Half dose $(12.5 \mathrm{mg} / \mathrm{kg})$ and full dose $(25 \mathrm{mg} / \mathrm{kg})$ of XSLJZD were administered to the rats in the low-dose and XSLJZD-treated groups, respectively. Domperidone (3 $\mathrm{mg} / \mathrm{kg}$ Xian Janssen Pharmaceutical Ltd.) was administered to the rats in the domperidone-treated group. The full dosage of XSLJZD administered to rats was converted from the dosage administered to humans. Meanwhile, the model and control groups received $5 \mathrm{~mL} / \mathrm{kg}$ water daily by oral gavage for 10 days.

\section{Food intake measurement}

Food intake was measured before and after the drug treatment. After $18 \mathrm{~h}$ of fasting, the rats were housed individually. The food was provided for $7 \mathrm{~h}$, and the food consumption was calculated.

\section{Sucrose preference test (SPT)}

SPT [29-31] was conducted before and after the drugs were administered to the rats. Before the test was performed, the rats were treated to adapt to sucrose solution. In the training session, the rats were housed individually for $48 \mathrm{~h}$ in a cage with two bottles; one bottle contained $1 \%$ sucrose solution, whereas the other bottle contained tap water. The bottles were placed to the left side and to the right side of the feeding compartment; the positions of these bottles were switched at an interval of $12 \mathrm{~h}$ to prevent possible effects of side preference in drinking behaviour. After the training session was completed, only tap water was provided for $6 \mathrm{~h}$. Food and water were then withheld from the rats for $18 \mathrm{~h}$. In the test session, the rats were provided access to two bottles containing $1 \%$ sucrose solution and water for 
Table 1 Components of the XSLJZD solution

\begin{tabular}{lll}
\hline Scientific name & Part used & Proportion of ingredients (100 \%) \\
\hline Astragalus mongholicus & Root & 12 \\
Codonopsis pilosula & Root & 12 \\
Rhizoma Atractylodis Macrocephalae & Rhizome & 12 \\
Poria cocos & Sclerotium & 12 \\
Fructus Aurantii & Fruit & 12 \\
Amomum villosum & Fruit & 6.4 \\
Ligusticum chuanxiong Hort. & Sclerotium & 9.6 \\
Rhizoma corydalis & Rhizoma & 9.6 \\
Medicated Leaven & Fermentation products & 12 \\
Glycyrrhiza uralensis Fisch. & Root & 2.4 \\
\hline
\end{tabular}

$1 \mathrm{~h}$. Sucrose preference (SP) was quantified with the following equation: $\mathrm{SP}=[$ sucrose intake $(\mathrm{g})] /[$ sucrose intake $(\mathrm{g})+$ water intake $(\mathrm{g})$ ]. The proportion of rats in each group with an SP value of $>75 \%$ was counted and compared through chi-square test.

\section{Gastric balloon distensions for electromyographic (EMG) testing [28]}

After being subjected to overnight fasting, the rats were anesthetised intraperitoneally with $1 \%$ pentobarbital sodium (3 mg/kg) after the drugs were administered for 10 days. Balloons $(2.5 \mathrm{~cm}$ in length) made from latex condoms were attached to a long catheter. An epigastric incision was made, and the balloon was placed in the stomach through an incision at the tip of the fundus. The pylorus was not obstructed, and no blockage of gastric emptying was observed. A polyethylene tubing to inflate

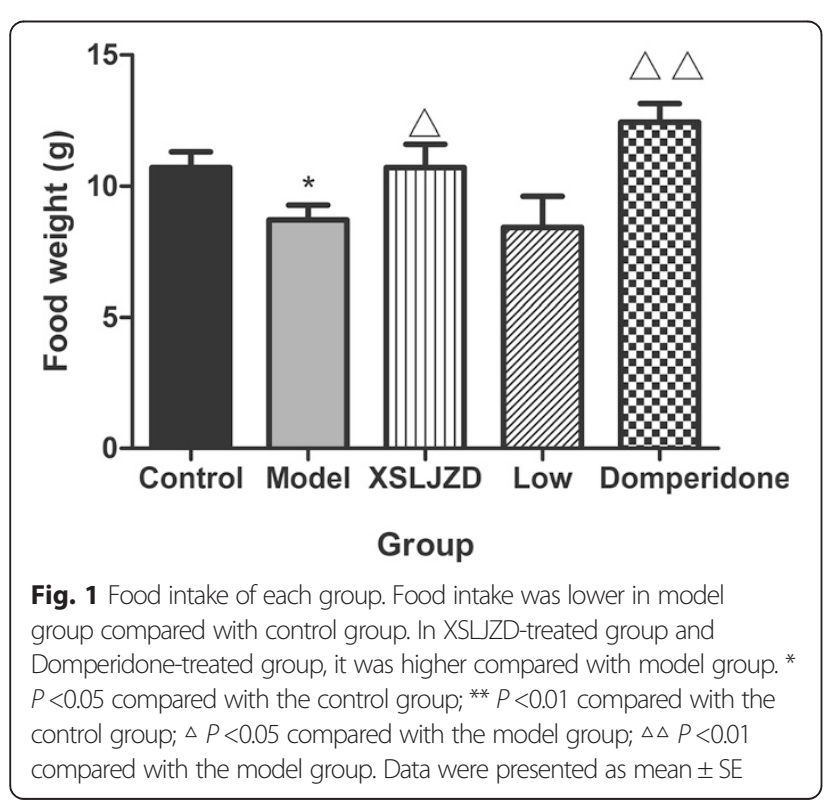

the gastric balloon with air was exteriorised at the back of the neck. Electromyographic (EMG) studies were conducted a week after surgery. Before the experiment, all the animals were anesthetised intraperitoneally with $1 \%$ pentobarbital sodium (3 $\mathrm{mg} / \mathrm{kg})$. Then, a pair of stainless steel wires was implanted into the acromiotrapezius (a superficial neck muscle) and externalised at the back of the neck for EMG recordings.

In the experiment, the rats received a series of $20 \mathrm{~s}$ gastric balloon distensions: 10, 20, 30, 40 and $50 \mathrm{mmHg}$ (measured with a sphygmomanometer) at an interval of $2 \mathrm{~min}$ between distensions. A BL-420S biological and functional experimental system was used to record EMG continuously and to visualise data. EMG was corrected, and the area under the curve was calculated for $20 \mathrm{~s}$ distension period. The baseline activity, obtained $20 \mathrm{~s}$ before distension, was subtracted from the EMG induced by distension. The data

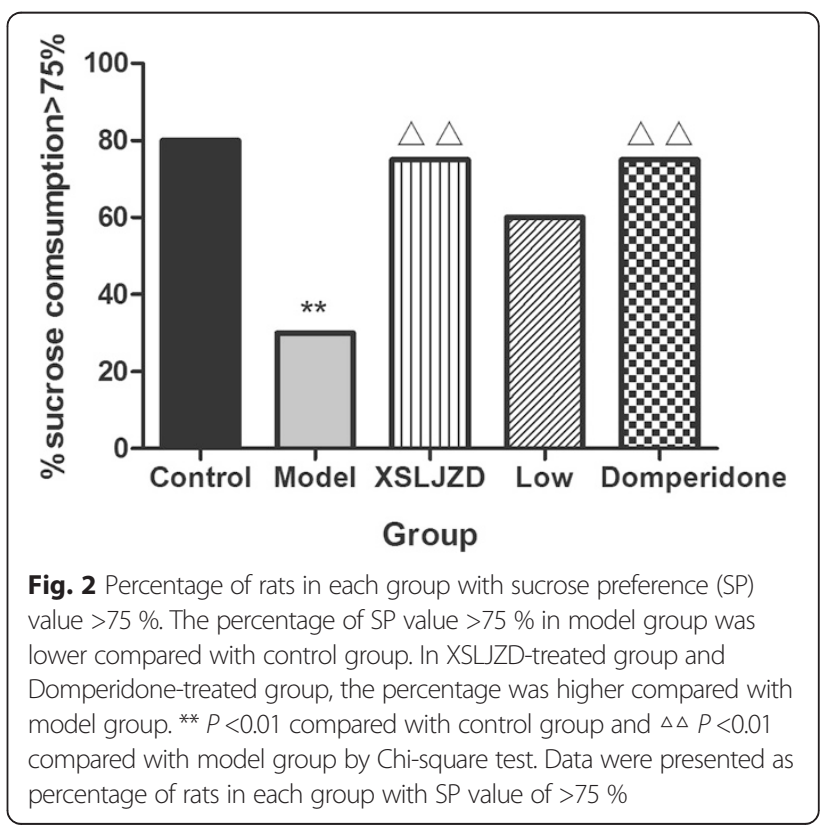




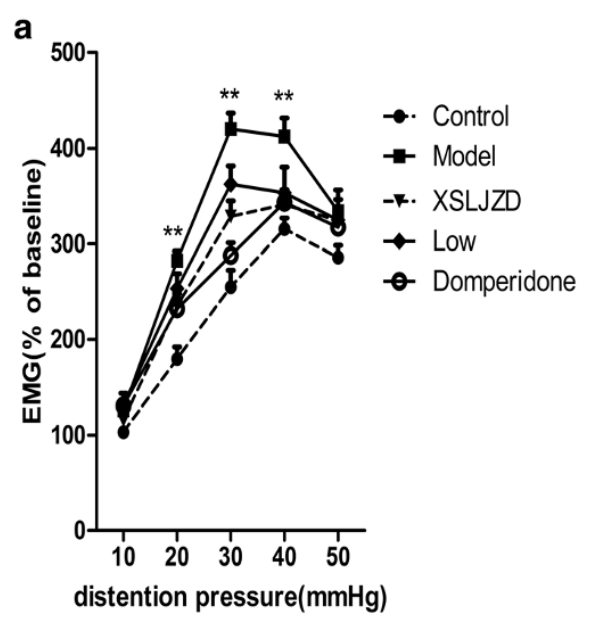

b Baseline

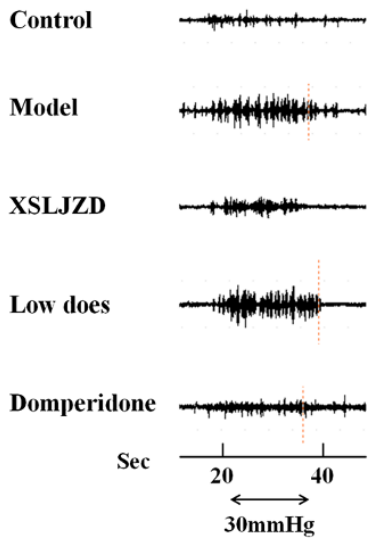

Fig. 3 Electromyographic (EMG) response to gastric distention of rats in each group. a EMG response to gastric distention of each group at distension of $10 \mathrm{mmHg}$ to $50 \mathrm{mmHg}$. In XSLZD-treated group, EMG was reduced significantly compared with model group at distension of $20 \mathrm{mmHg}$, $30 \mathrm{mmHg}$ and $40 \mathrm{mmHg} .{ }^{* *} P<0.01$ compared with control group. Data were presented as mean \pm SE. b Representative EMG response at $30 \mathrm{mmHg}$ of each group
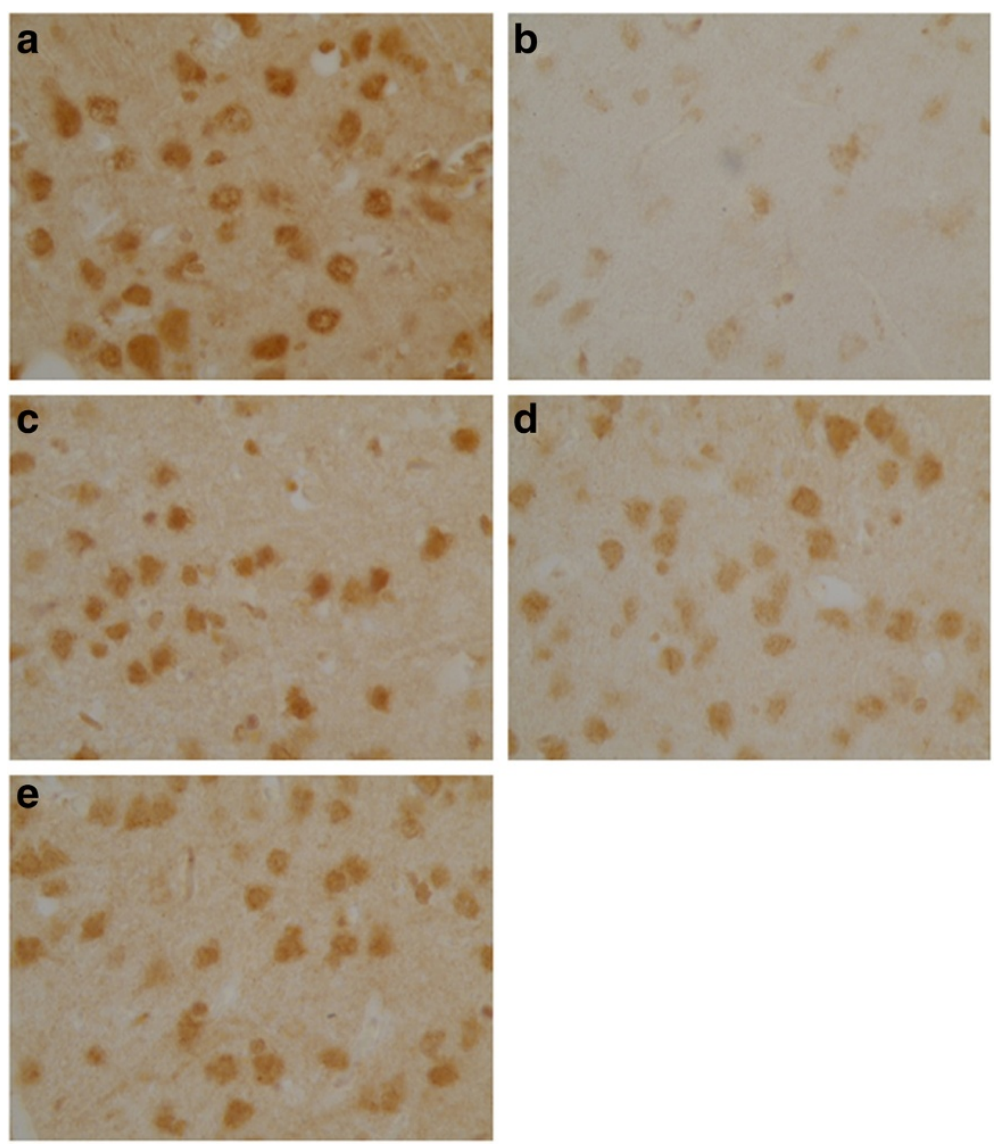

Fig. 4 Expression of Ghrelin in hypothalamus of each group. a Control group. b Model group. c XSLZD-treated group. $\mathbf{d}$ Low-dose XSLZD treated group. e Domperidone-treated group. Ghrelin distributed in cytoplasm of hypothalamus. (Tissue sections were viewed at 100x magnification.) Positive cells for Ghrelin were brown and circular or pear-shaped. Fewer positive cells can be seen in the model group 
were presented as the change from the baseline as a function of distension pressure.

\section{Immunohistochemistry}

After 10 days of drug treatment, the rats were anesthetised with $1 \%$ pentobarbital sodium. Brains and stomachs were removed, fixed with $10 \%$ formalin and embedded in paraffin. The tissues were subsequently cut in $10 \mu \mathrm{m}$ sections, mounted on Superfrost Plus slides (1 section/slide) and stored at room temperature. Before the experiment was conducted, the slides were deparaffinised using xylene, subjected to microwave heat-mediated antigen retrieval using citrate buffer at $\mathrm{pH} 6$ for $45 \mathrm{~min}$ and were cooled to room temperature. The specimens were immersed in $3 \% \mathrm{H}_{2} \mathrm{O}_{2}$ for $20 \mathrm{~min}$ to inactivate endogenous peroxidase and were subsequently washed with PBS (thrice for $2 \mathrm{~min}$ each). The sections were incubated overnight at $4{ }^{\circ} \mathrm{C}$ with the following antibodies: polyclonal rabbit anti-ghrelin (1:100, Abbiotec), polyclonal rabbit anti-VIP (1:20, Abcam) and polyclonal rabbit antiCCK-8 (1:100, Abbiotec) in buffer containing $0.01 \mathrm{M}$
PBS. By 9:00 a.m. the next day, the slides were washed with PBS (thrice for 2 min each) and were incubated in Polink-2 Plus ${ }^{\ominus}$ Polymer HRP Detection System (ZSGB-BIO, Beijing) according to the manufacturer's instructions. Afterwards, the sections were washed thrice with PBS, visualised by using DAB for $10 \mathrm{~min}$ at $37{ }^{\circ} \mathrm{C}$ and rinsed with running water for 2 min. After dehydration, the slides were mounted using neutral balsam. The sections were visualised under a microscope, and images were acquired using a camera. At least three sections per rat and three rats per group were analysed. Mean integrated optical density (MOD) was calculated using Image-Pro Plus version 6.0 analysis software.

\section{Real-time PCR}

After 10 days of drug treatment, the rats were anesthetised with $10 \%$ chloral hydrate. The hypothalamus and stomachs of these rats were removed. mRNA was quantified by using quantitative RT-PCR. Total RNA was extracted from the rats' hypothalamus and stomach by using Promega SV total RNA isolation system (Promega, USA) according to the
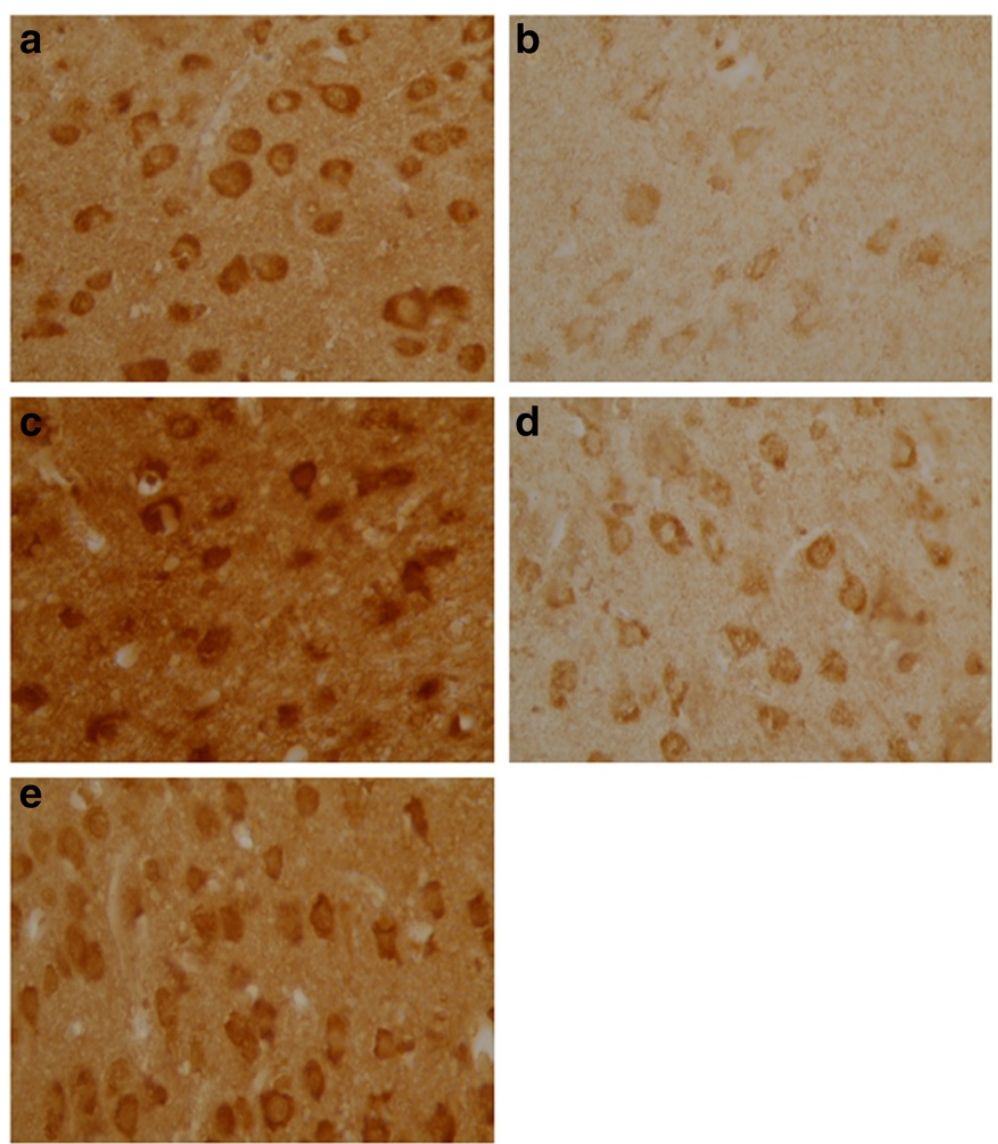

Fig. 5 Expression of CCK-8 in hypothalamus of each group. a Control group. b Model group. c XSLZD-treated group. $\mathbf{d}$ Low-dose XSLZD treated group. e Domperidone-treated group. CCK-8 mainly distributes in cytoplasm of hypothalamus. (Tissue sections were viewed at 100x magnification.) Positive cells for CCK-8 were brown and circular or oval-shaped. Fewer positive cells can be seen in the model group and low-dose XSLZD-treated group 
manufacturer's instructions. Total RNA $(2 \mu \mathrm{g})$ was reversetranscribed by using PromegaGoScript (Promega, USA) according to the manufacturer's instructions. The thermocycling conditions used are listed as follows: initial activation at $95{ }^{\circ} \mathrm{C}$ for $30 \mathrm{~s} ; 40$ cycles of denaturation at $95^{\circ} \mathrm{C}$ for $5 \mathrm{~s}$, annealing at $60{ }^{\circ} \mathrm{C}$ for $30 \mathrm{~s}$; and melt curve determination at $65{ }^{\circ} \mathrm{C}$ to $95{ }^{\circ} \mathrm{C}$ for $5 \mathrm{~s}$. Primers were designed using Primer-BLAST (NCBI, USA) according to the mRNA sequences (by GenBank) of ghrelin (NM_021669.2), VIP (NM_053991.1), CCK (NM_012829.2) and glyceraldehyde3-phosphate dehydrogenase (GAPDH; NM_017008.4, as control). The PCR products were run on $0.8 \%$ agarose gel to confirm that these products were of the expected size. Results were normalised against GAPDH expression. The primer sequences are listed as follows. The forward and reverse primers of the GAPDH gene were $5^{\prime}$-GGCACAGT CAAGGCTGAGAATG-3' and 5' -ATGGTGGTGAAGAC GCCAGTA-3', respectively. The forward and reverse primers of the ghrelin gene were $5^{\prime}$-CCAAGGCCAT GGTGTCTTCA-3' and 5'-CTGCAGTTTAGCTGG TGGCTTC-3', respectively. The forward and reverse primers of the VIP gene were $5^{\prime}$-TCAGTTCCT GGCGATCCTGAC-3' and 5' -CTCCGCTAAGGCAT TCTGCAA-3', respectively. The forward and reverse primers of the CCK gene were 5'-CCCGATACATC CAGCAGGTC-3' and 5'-AAATCCATCCAGCCCA TGTAGTC-3', respectively. $2^{-\Delta \Delta C t}$ was calculated, and differences between groups were analysed by using non-parametric tests.

\section{ELISA}

Rats were anesthetised with $10 \%$ chloral hydrate after 10 days of drug treatment. Blood samples were collected in blank sterile tubes and allowed to clot for $2 \mathrm{~h}$ at room temperature. Subsequently, these samples were centrifuged at $1000 \mathrm{rpm}$ for $15 \mathrm{~min}$. Serum was removed and stored at $-80{ }^{\circ} \mathrm{C}$. Ghrelin, VIP, and CCK were quantified using specific ELISA kits supplied by CUSABIO (Wuhan, China). Each serum $(100 \mu \mathrm{L})$ was mixed with sample diluent according to the manufacturer's instructions. Absorbance was determined at $450 \mathrm{~nm}$.
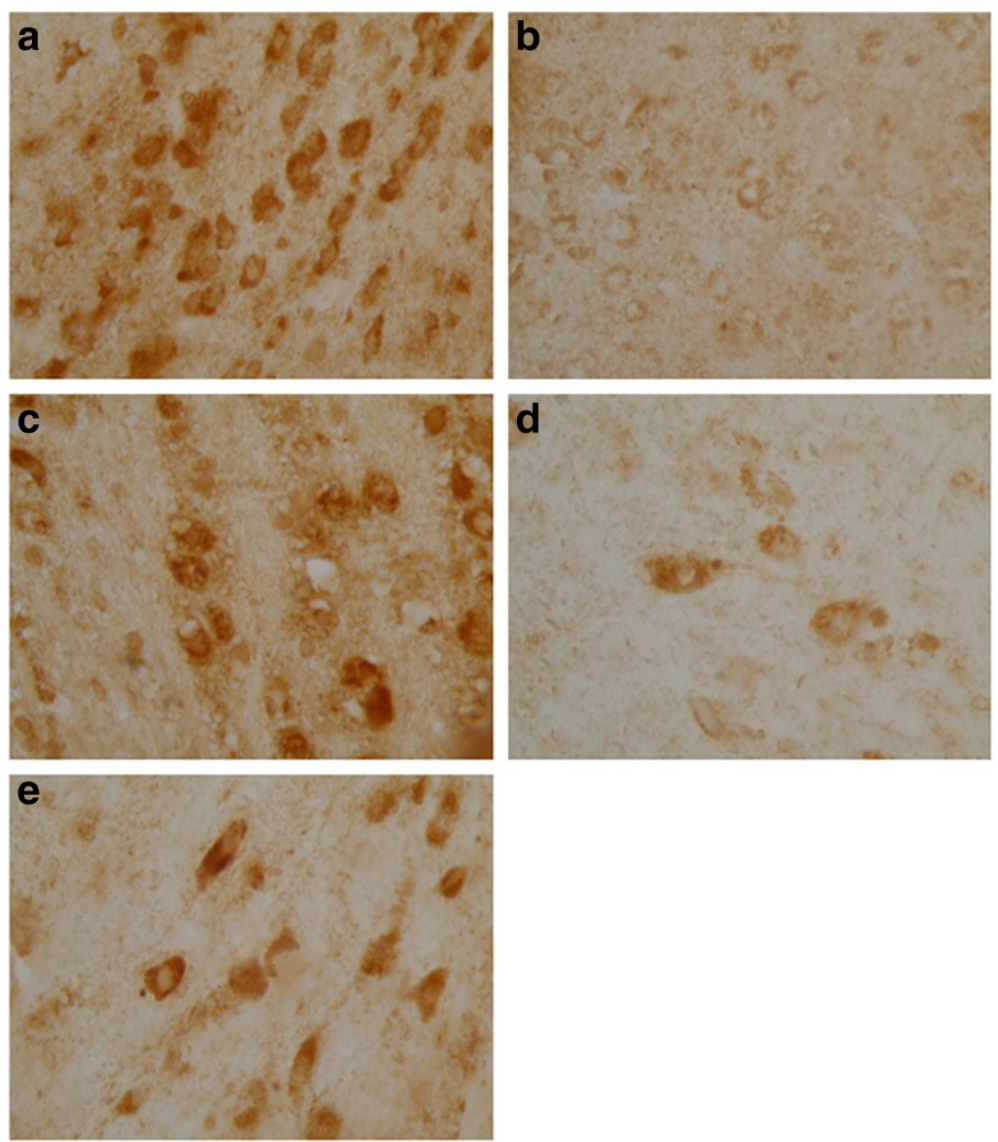

Fig. 6 Expression of VIP in hypothalamus of each group. a Control group. b Model group. c XSLJZD-treated group. d Low-dose XSLJZD treated group. e Domperidone-treated group. Neuropepide VIP mainly distributes in cytoplasm of hypothalamus. (Tissue sections were viewed at 100X magnification.) Positive cells for VIP were brown and circular or oval-shaped. Fewer positive cells can be seen in the model group and low-dose XSLIZD-treated group and domperidone-treated group 


\section{Data analysis}

All values, except those obtained by sucrose preference test, were presented as means \pm SE. One-way ANOVA or non-parametric test was conducted for comparison. Post hoc comparisons were performed using Student-Newman-Keuls test or Mann-Whitney $U$ test. Statistical analysis was performed in SPSS 17.0. $P<0.05$ was considered statistically significant.

\section{Results}

XSLJZD increased the food intake of the rats with FD

After overnight fasting, rats with FD consumed lesser amount of food than the control rats during a $7 \mathrm{~h}$ period ( $10.7 \pm 0.6$ vs. $8.7 \pm 0.5 ; P=0.01 ; n=10$ in each group). The XSLJZD-treated group consumed greater amount of food than the model group $(10.7 \pm 0.9$ vs. $8.7 \pm 0.5 ; P=0.04 ; n=$ 10 in each group). Likewise, thedomperidone-treated group consumed greater amount of food than the model group $(12.4 \pm 0.7$ vs. $8.7 \pm 0.5 ; P=0.00)$. No significant difference was observed between the low-dose XSLJZD-treated group and the model group $(8.4 \pm 1.2$ vs. $8.7 \pm 0.5 ; P>0.1)$ (Fig. 1$)$.
XSLZZ increased the percentage of sucrose consumption (>75\%) of rats with FD

In the sucrose preference test, no significant difference was observed in terms of sucrose and water intake among the groups $(P>0.05)$. However, the percentage of rats with SP value of $>75 \%$ was significantly reduced in rats with FD (30\%) compared with the control rats (80\%; $P=0.001$; $n=10$ in each group), as indicated by chi-square test. The percentage significantly increased in the XSLJZD-treated group (75 \%) and in the domperidone-treated group (75\%) compared with the rats with FD $(30 \% ; P=0.004 ; n=10$ in each group). The low-dose XSLJZD-treated group did not exhibit significant difference from the model group (Fig. 2).

\section{XSLJZD reduced the hypersensitivity to gastric distention of rats with FD}

After the 10-day treatment, EMS testing was performed. Compared with the control rats, rats with FD significantly increased in EMG at distension pressures of $20 \mathrm{mmHg}(179.3 \%$ vs. $282.5 \% ; P=0.000 ; n=3$ in each group), $30 \mathrm{mmHg}(254.9 \%$ vs. $420.1 \% ; P=$
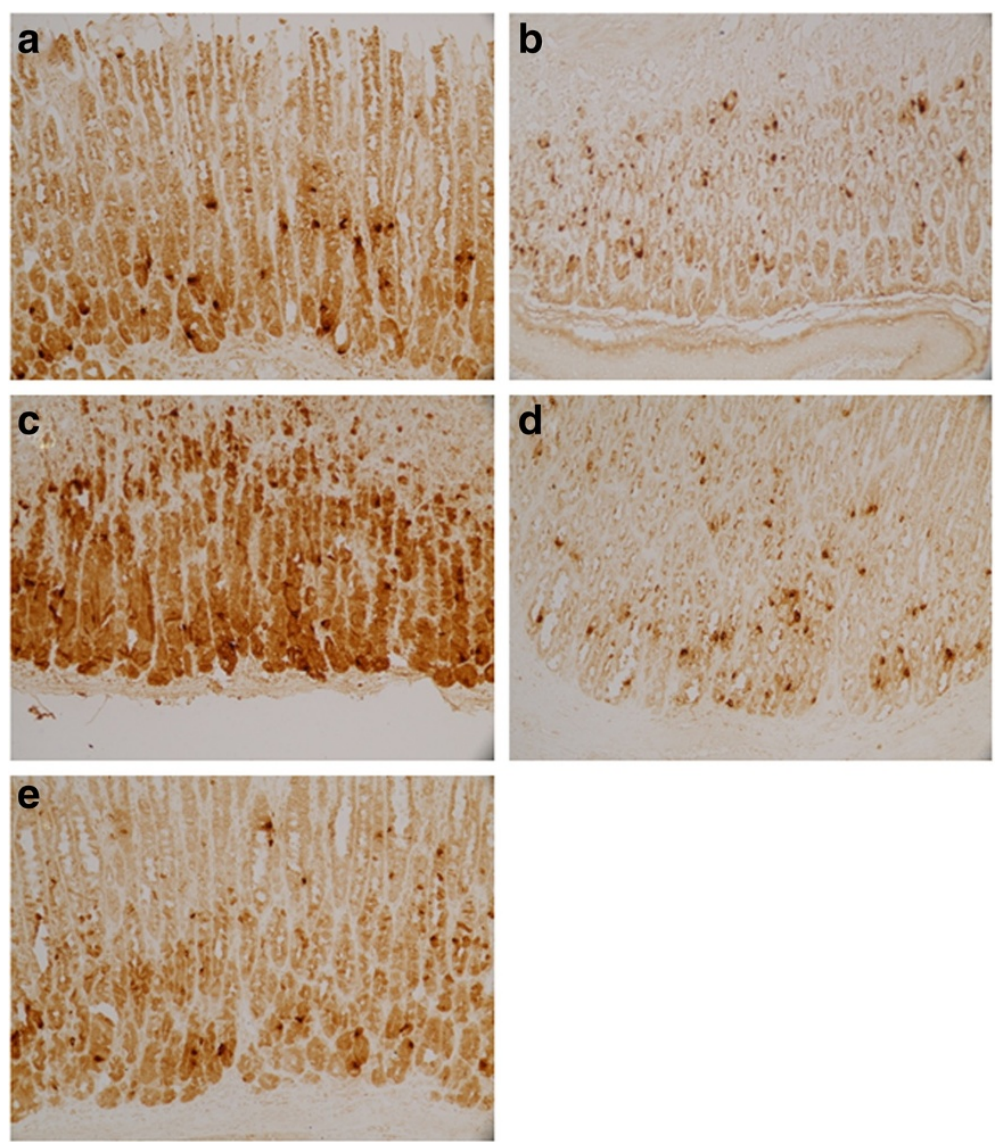

Fig. 7 Expression of Ghrelin in stomach of each group. a Control group. b Model group. c XSபZD-treated group. d Low-dose XSLZD treated group. e Domperidone-treated group. Ghrelin was expressed as granular neuropetide in the cytoplasm of the stratum basale of the stomach (Tissue sections were viewed at 20x magnification.) Brown cells were positive for Ghrelin. Model group expressed lower Ghrelin than control group, XSLZD significantly increased ghrelin of rats with FD 
$0.000)$ and $40 \mathrm{mmHg}(315.4 \%$ vs. $412.3 \% ; P=0.002)$. However, no significant difference was observed at $50 \mathrm{mmHg}$. XSLJZD inhibited the EMG activity of rats with FD to gastric distension in a dose-dependent manner. XSLJZD elicited a significant effect at 20 $(277.2 \%$ vs. $282.5 \% ; \quad P=0.016), 30 \quad(398.3 \%$ vs. $420.1 \% ; \quad P=0.003)$ and $40 \mathrm{mmHg}(405.5 \%$ vs. $412.3 \% ; P=0.015)$. Low dose significantly affected EMG responses only at $30(362.4 \%$ vs. $420.1 \% ; P=$ 0.034 ) and $40 \mathrm{mmHg}$ ( $353.3 \%$ vs. $412.3 \%$; $P=0.038$ ) compared with the rats with FD, as indicated by oneway ANOVA. Furthermore, the control group did not significantly differ from the domperidone-treatment group $(P>0.1)$ (Fig. 3$)$.

\section{Expression of relative neuropeptides in the brain and the stomach of each group}

Ghrelin, CCK-8 and VIP were expressed as granular neuropeptides in the cytoplasm of the hypothalamus (Figs. 4, 5 and 6) and the stratum basale of the stomach (Figs. 7, 8 and 9). The expressions of these neuropeptides were lower in the brain and in the stomach of rats with FD compared with those of the control rats. In the stomach and the hypothalamus, ghrelin, CCK- 8 and VIP of the rats with FD were lower than those of the control rats $(P<0.05 ; n=3$ in each group). Low-dose XSLJZD-treated groups did not significantly differ from the model group. However, XSLJZD significantly increased ghrelin, CCK-8 and VIP of rats with FD $(P<0.05 ; n=3)$. Ghrelin, CCK- 8 and VIP of the domperidone-treated group were also higher than those of the model group, except for VIP in the hypothalamus $(P<0.05 ; n=3)$ (Table 2 and Fig. 10).

\section{XSLZD increased the neuropeptides in the serum of rats with FD}

Ghrelin was reduced in the serum of rats with FD compared with that of the control rats $(46.72 \pm 4.92$ vs. $189.9 \pm 49.96 ; P=0.007 ; n=7$ in each group). By contrast, ghrelin was increased in the XSLJZDtreated group compared with the model group
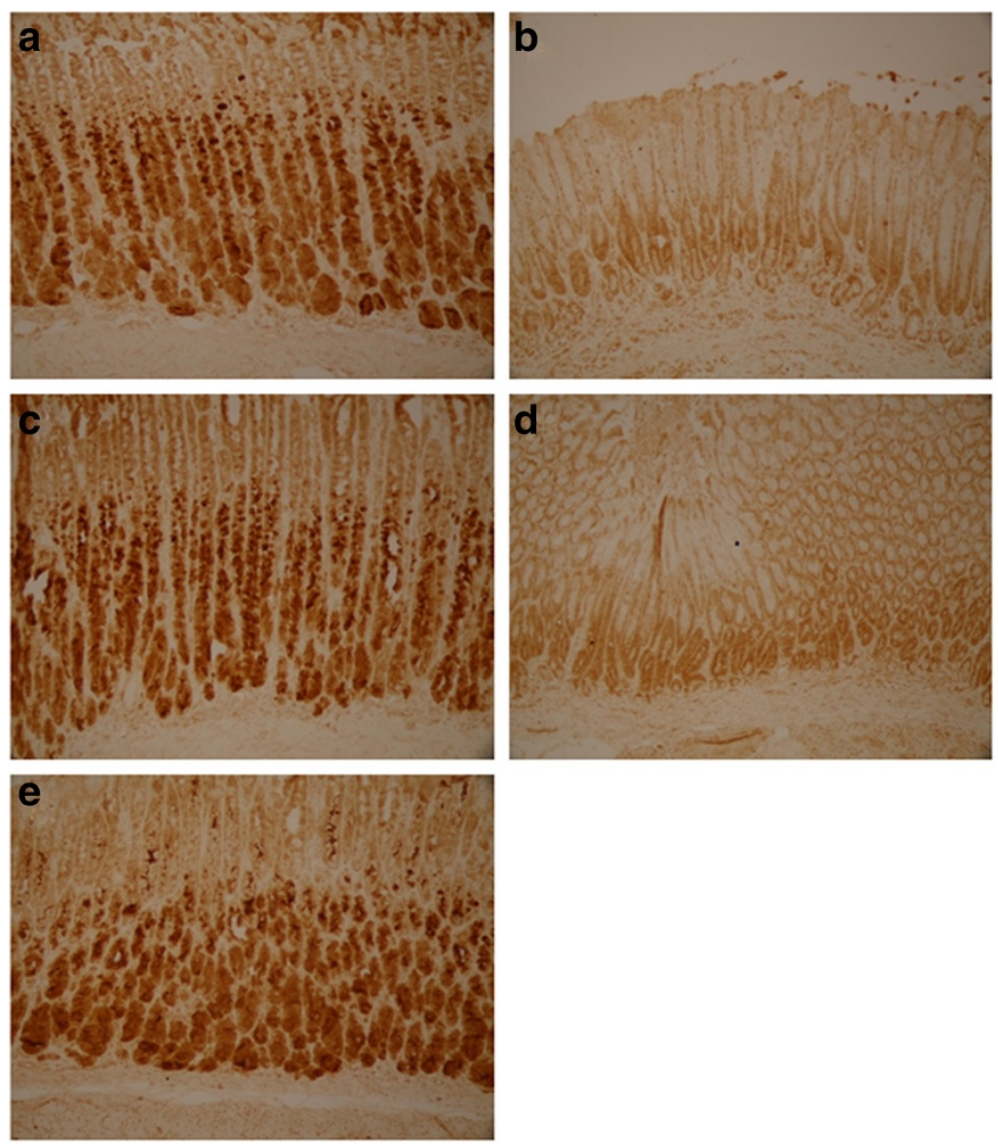

Fig. 8 Expression of CCK-8 in stomach of each group. a Control group. b Model group. c XSLZD-treated group. d Low-dose XSLZD treated group. e Domperidone-treated group. CCK-8 mainly distributes in stratum basale of stomach. (Tissue sections were viewed at 20X magnification.) Positive CCK-8 expressed as brown granule, distributed in cytoplasm. Expression of CCK-8 was lower in model group and low-dose XSLZD-treated group, higher in control group, XSLZD-treated group and domperidone-treated group 

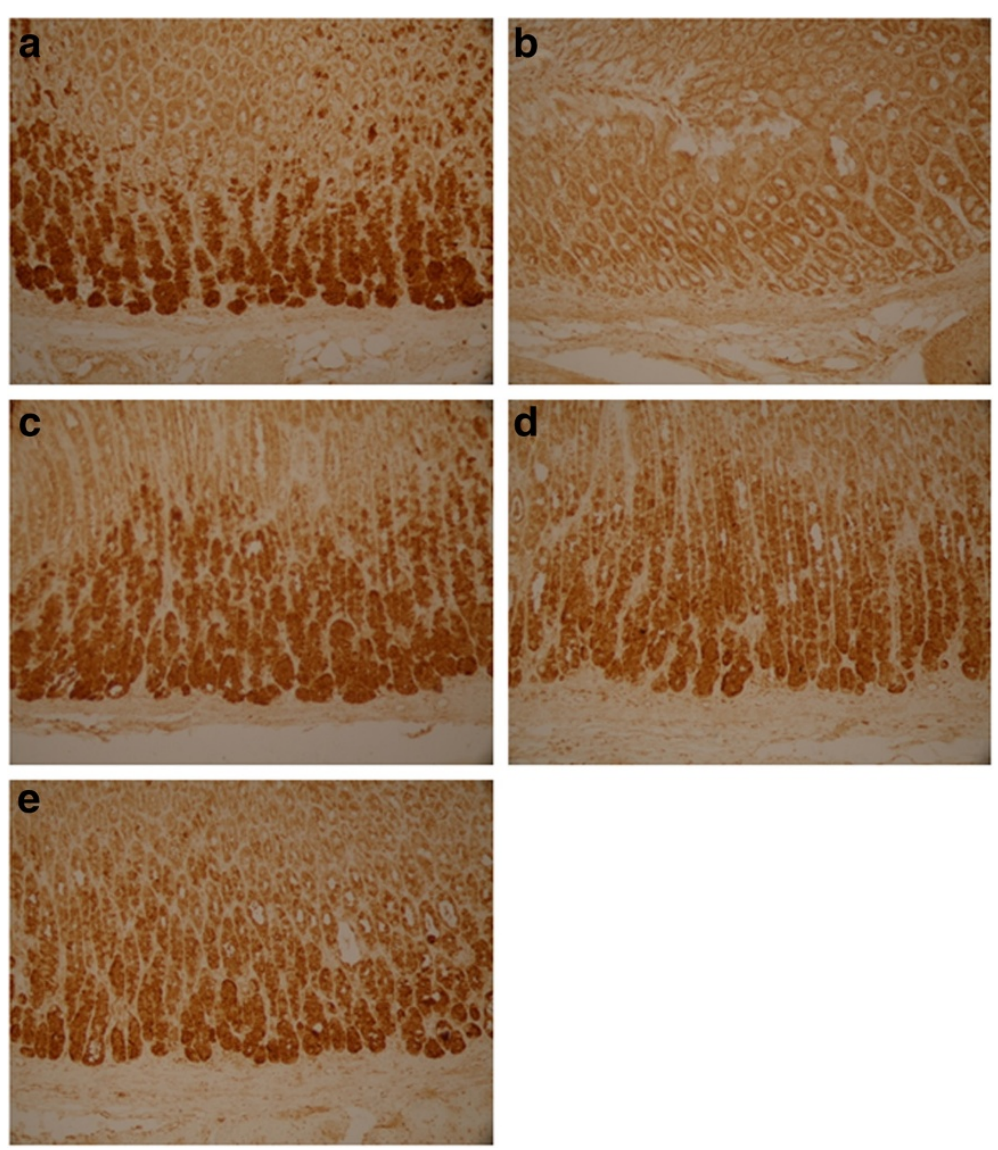

Fig. 9 Expression of VIP in stomach of each group. a Control group. b Model group. c XSLJZD-treated group. d Low-dose XSLJZD treated group. e Domperidone-treated group. VIP was expressed as granular neuropetide in the cytoplasm of the stratum basale of the stomach (Tissue sections were viewed at 20x magnification.) Brown cells were positive for VIP. Expression of VIP was lower in model group and higher in control group, XSLJZD-treated group and domperidone-treated group

$(186.4 \pm 40.13$ vs. $46.72 \pm 4.92 ; P=0.009 ; n=7$ in each group). No significant difference was observed among the control group, low-dose XSLJZD-treated group and domperidone-treated group in terms of ghrelin in the serum. Similar to ghrelin, CCK was reduced in the serum of rats with FD compared with that of the control rats $(22.77 \pm 3.59$ vs. $77.19 \pm 14.36 ; P=0.003$; $n=7$ in each group). CCK was increased by XSLJZD in a dose-dependent manner. The low-dose XSLJZD-treated groups did not significantly differ with the rats with FD in

Table 2 MOD value of relative neuropeptides in the stomach and hypothalamus of each group

\begin{tabular}{lllll}
\hline Part & Groups & Ghrelin & CCK-8 & VIP \\
\hline Stomach & Control & $0.583 \pm 0.008$ & $0.714 \pm 0.042$ & $0.823 \pm 0.025$ \\
& Model & $0.368 \pm 0.010^{* *}$ & $0.467 \pm 0.057^{*}$ & $0.486 \pm 0.025^{* *}$ \\
& XSLJZD & $0.716 \pm 0.050^{* \Delta}$ & $0.706 \pm 0.090^{\Delta}$ & $0.861 \pm 0.107^{\Delta \Delta}$ \\
& Low dose XSLJZD & $0.356 \pm 0.044^{* *}$ & $0.498 \pm 0.039$ & $0.652 \pm 0.082$ \\
Dypothalamus & Domperidone & $0.543 \pm 0.163^{\Delta \Delta}$ & $0.863 \pm 0.122^{\Delta \Delta}$ & $0.711 \pm 0.092^{\Delta}$ \\
& Control & $0.497 \pm 0.036$ & $0.825 \pm 0.081$ & $0.561 \pm 0.077$ \\
& Model & $0.268 \pm 0.010^{* *}$ & $0.372 \pm 0.006^{* *}$ & $0.365 \pm 0.017^{*}$ \\
& XSLJZD & $0.417 \pm 0.017^{\Delta \Delta}$ & $0.995 \pm 0.088^{\Delta \Delta}$ & $0.594 \pm 0.105^{\Delta}$ \\
& Low dose XSLJZD & $0.372 \pm 0.011^{* \Delta}$ & $0.540 \pm 0.050^{*}$ & $0.391 \pm 0.012$ \\
& Domperidone & $0.441 \pm 0.059^{\Delta \Delta}$ & $0.824 \pm 0.163^{\Delta \Delta}$ & $0.449 \pm 0.046$ \\
\hline
\end{tabular}



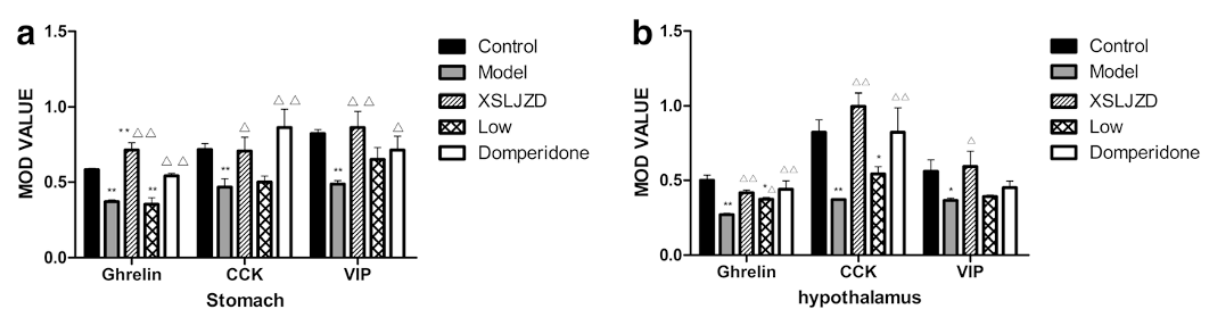

Fig. 10 Mean integrated optical density (MOD) value of relative neuropeptides. a MOD value of neuropeptides in the stomach. $\mathbf{b}$ MOD value of neuropeptides in hypothalamus. * $P<0.05$ compared with control group; ${ }^{*} P<0.01$ compared with control group. $\Delta P<0.05$ compared with the model group; $\Delta \Delta P<0.01$ compared with the model group. Data were presented as mean $\pm \mathrm{SE}$

terms of CCK in the serum $(P>0.10)$; however, the XSLJZD-treated group exhibited a significant increase in serum CCK compared with that of rats with FD $(82.97 \pm$ 13.47 vs. $22.77 \pm 3.59 ; P=0.001 ; n=7$ in each group). VIP was reduced in the serum of rats with FD $(16.95 \pm 5.15$ vs. $75.61 \pm 20.12 ; P=0.003 ; n=7$ in each group) compared with that in the control group. VIP in the XSLJZD-treated group significantly increased compared with that in the model group $(62.71 \pm 19.05$ vs. $16.95 \pm 5.15 ; P=0.017 ; n=$ 7 in each group). No significant difference in VIP of lowdose XSLJZD-treated groups and domperidone-treated groups was observed. Nevertheless, a moderate dose of XSLJZD could increase VIP in the serum (Fig. 11).

\section{XSLZD increased the gene expression of the relative} neuropeptides in the stomach

In the stomach, the mRNA expressions of ghrelin, CCK and VIP of rats with FD were lower compared with those of the control rats $(P<0.05 ; n=6$ in each group). The mRNA expressions of CCK and VIP of the XSLJZD-treated group were higher than those of the model group. No significant difference in ghrelin expression was observed in the model and drug-treated groups. Nevertheless, moderate-dose XSLJZD increased ghrelin mRNA expression in the stomach. In the hypothalamus, the mRNA expressions of ghrelin, CCK and VIP of rats with FD were higher than those of the control rats $(P<0.05 ; n=6$ in each group). The mRNA expression of ghrelin in the XSLJZD-treated and lowdose XSLJZD-treated groups as well as in the domperidone-treated group was reduced compared with that of the model group $(P<0.05)$. CCK mRNA expression in the low-dose XSLJZD-treated group and domperidone-treated group was lower $(P<0.05)$ than that of the model group. Furthermore, VIP mRNA expression in thedomperidone-treated group was
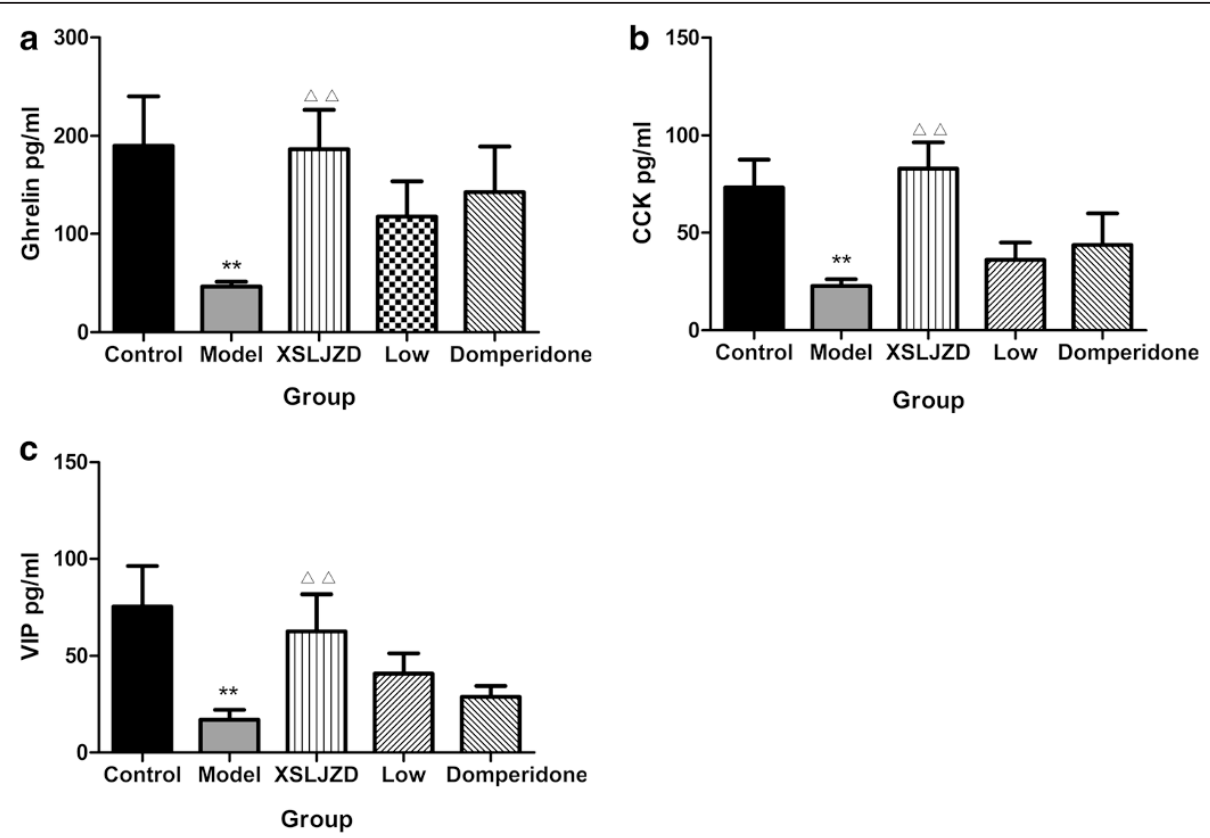

Fig. 11 Expression of relative neuropeptides in the serum. a Expression of Ghrelin of each group. $\mathbf{b}$ Expression of CCK of each group. $\mathbf{c}$ Expression of VIP of each group. ${ }^{* *} P<0.01$ compared with the control group; $\triangle \triangle P<0.01$ compared with the model group. Data were presented as mean $\pm S E$ 
Table 3 The mRNA expression of relative nouropeptides in the stomach and hypothalamus of each group

\begin{tabular}{|c|c|c|c|c|}
\hline Part & Groups & Ghrelin & CCK & VIP \\
\hline \multirow[t]{5}{*}{ Stomach } & Control & $1.536 \pm 0.509$ & $0.981 \pm 0.069$ & $1.092 \pm 0.244$ \\
\hline & Model & $0.232 \pm 0.172^{*}$ & $0.291 \pm 0.121^{*}$ & $0.111 \pm 0.059^{*}$ \\
\hline & XSLJZD & $1.735 \pm 0.747$ & $1.212 \pm 0.320^{\Delta \Delta}$ & $1.127 \pm 0.336^{\Delta}$ \\
\hline & Low dose XSLJZD & $0.439 \pm 0.074$ & $0.428 \pm 0.125$ & $0.394 \pm 0.139$ \\
\hline & Domperidone & $1.797 \pm 0.869$ & $0.637 \pm 0.095$ & $0.555 \pm 0.417$ \\
\hline \multirow[t]{5}{*}{ hypothalamus } & Control & $0.967 \pm 0.172$ & $1.075 \pm 0.268$ & $0.871 \pm 0.222$ \\
\hline & Model & $4.917 \pm 0.239^{*}$ & $2.923 \pm 0.640^{*}$ & $2.084 \pm 0.442^{*}$ \\
\hline & XSLJZD & $2.646 \pm 0.797^{\triangle}$ & $2.162 \pm 0.511^{*}$ & $0.777 \pm 0.295$ \\
\hline & Low dose XSLJZD & $2.804 \pm 0.407^{\triangle}$ & $0.916 \pm 0.281^{\Delta}$ & $1.268 \pm 0.285$ \\
\hline & Domperidone & $1.925 \pm 0.448^{\triangle}$ & $1.113 \pm 0.247^{\Delta}$ & $0.617 \pm 0.073^{\Delta}$ \\
\hline
\end{tabular}

${ }^{*} P<0.05$ compared with the control group; ${ }^{\Delta} P<0.05$ compared with the model group; ${ }^{\Delta \Delta} P<0.01$ compared with the model group

reduced $(P<0.05)$ compared with that of the model group. The mRNA expression of these neuropeptides was not altered in a dose-dependent manner. However, the mRNA expressions of ghrelin, CCK and VIP in the hypothalamus of rats with FD were reduced by XSLJZD (Table 3 and Fig. 12).

\section{Discussion}

Our findings demonstrated the mechanism by which the Chinese medicine XSLJZD relieved FD. Previous studies revealed that FD is associated with gastric hypersensitivity to mechanical distention and impaired accommodation $[28,32]$. Gastric hypersensitivity or motor change can be caused by impairment, such as gastric inflammation or mechanical impairment, which can occur during childhood; the change remains, but the impairment was resolved completely [33, 34]. Previous studies showed that iodoacetamidecan induce mild damage to the gastric surface and chronically sensitise gastric sensory afferents during adulthood in the absence of overt structural abnormalities. A rat model displayed the symptoms of functional dyspepsia similar to that experienced by human beings [28]. Therefore, iodoacetamide-treated rats as FD model are used to study the effects and mechanisms of Chinese medicine XSLJZD.

Studies provided insights into the biological basis of this form of neonatal programming on the brain-gut axis [10], and they also demonstrated the release of neuropeptides such as ghrelin, CCK and VIP. Ghrelin, CCK and VIP are produced in the stomach and then released into the circulation $[14,20,25]$. The arcuate nucleus (ARC) of the hypothalamus is implicated in food intake regulation and energy homeostasis. ARC is adjacent to the median eminence, which is a 'circumventricular organ' with fenestrated capillaries and an incomplete blood-brain barrier [35]. Circulating gut hormones can pass across the median eminence and directly affect the activity of ARC neurons. The hypothalamus receives both hormonal signals and peripheral neurons, and then relay information regarding energy availability [36]. Hormonal signals from the periphery are integrated with high brain centre signals to regulate appetite and control energy expenditure. The release of gut hormones, such as ghrelin, CCK and VIP, regulates the brain-gut axis using hormonal signals [16, 17, 20, 26].

In our study, XSLJZD could increase the food intake of FD rats. A decrease in sucrose solution consumption
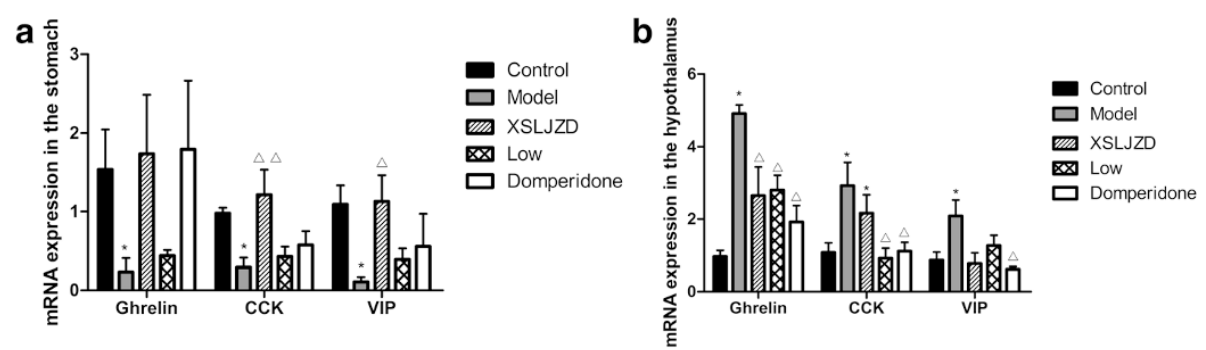

Fig. 12 mRNA expression of neuropeptides in the stomach (a) and hypothalamus (b) of each group. ${ }^{*} P<0.05$ compared with the control group; ${ }^{*} P<0.01$ compared with the control group; $\triangle P<0.05$ compared with the model group; $\triangle \triangle P<0.01$ compared with the model group by nonparametric test. Data were presented as mean $\pm \mathrm{SE}$ 
manifests anhedonia, which is the decreased ability to experience pleasures [30,37], because of its association with drink and food intake; this decrease also reflects the appetite of rats. In our experiment, model rats showed low percentage of SP value at $>75 \%$; XSLJZDinduced increase in SP percentage corresponded to the improvement of the appetite of rats with FD. Early satiation or postprandial fullness or pain in the upper gastrointestinal origin is the prominent feature observed in FD patients. Those symptoms in animals are conceptually manifested as gastric hypersensitivity to mechanical distention in the absence of overt morphological or histological changes. In FD patients, the presence of visceral hypersensitivity to graded gastric distention is possibly correlated with postprandial pain $[38,39]$; other non-painful symptoms, such as nausea, satiety and fullness, are also triggered at lower distension pressures. This result suggests that multimodal pathways are sensitized [40]. We reproduced this hypersensitivity in our experiment [28, 32, 41] and calculated the EMG to graded gastric distention at pressures ranging from $10 \mathrm{mmHg}$ to $50 \mathrm{mmHg}$. Rats with FD displayed significantly higher EMG in response to gastric distention at 20, 30 and $40 \mathrm{mmHg}$. Our results also showed that XSLJZD inhibited visceromotor responses caused at distention pressures of 30 and $40 \mathrm{mmHg}$. Previous studies also demonstrated that neonatal IA-treated rats display significantly higher behavioural scores in response to gastric distension at 40, 60 and $80 \mathrm{mmHg}$ [28]. In another study, rats with FD exhibit a significantly higher EMG in response to gastric distension at $50 \mathrm{mmHg}$ to $120 \mathrm{mmHg}$ with the same result [33]. This difference may be attributed to the instrument used in this study. We observed a dose-dependent effect of XSLJZD on food intake, sucrose preference test and response to mechanical gastric distension. Low dose elicited slight or no effect, and XSLJZD caused a more robust effect. According to the Rome III criteria, epigastric pain or buming, postprandial fullness and early satiation are symptoms of FD [42]. XSLJZD could alleviate the main symptoms of FD in our experiment.

In the experiment, transient gastric irritation in the neonatal period could result in impaired brain-gut axis and decrease some neuropeptides. Result manifested the strong association between FD and the brain-gut axis.

We also examined the effects of transient neonatal gastric inflammation on ghrelin, CCK and VIP expressions; the brain-gut axis response to stress; and the mechanism of XSLJZD on FD. The expressions of ghrelin, CCK and VIP in the stomach and in the serum were significantly lower in IA-treated rats compared with those in control rats. These neuropeptides were significantly increased by XSLJZD. The effect of XSLJZD on the neuropeptides was dose dependent. In particular, XSLJZD was more effective than the low dose. The expression of ghrelin, CCK and VIP were also significantly decreased in the hypothalamus of rats with FD compared with those of the control rats. However, the mRNA expressions of these neuropeptides were increased. XSLJZD increased the protein expression but decreased the mRNA expression of these neuropeptides in the hypothalamus. Ghrelin, CCK and VIP were produced in the stomach and then released into the blood $[14,20,25]$. In our experiment, XSLJZD could increase the production of these neuropeptides, thereby increasing the neuropeptide levels in circulation. The decrease in the mRNA expression of these neuropeptides in the hypothalamus maybe considered as feedback regulation.

\section{Conclusions}

Our results demonstrated that rats with FD with transient gastric irritation in the neonatal period showed brain-gut axis impairment and low neuropeptide level. Chinese medicine XSLJZD could alleviate the symptoms of FD and upregulate the brain-gut axis by increasing the production of neuropeptides such as ghrelin, CCK, and VIP. These findings have major implications in the mechanism of XSLJZD to relieve FD, which would help investigate FD mechanism and pharmacokinetics.

\section{Abbreviations}

XSLIZD: Xiangsha Liujunzi decoction; FD: Functional dyspepsia; CCK: Cholecystokinin; VIP: Vasoactive intestinal polypeptide;

GIT: Gastrointestinal tract; CNS: Central nervous system; ENS: Enteric nervous system; SP: Sucrose preference; EMG: Electromyographic; MOD: Mean integrated optical density; ARC: Arcuate nucleus.

\section{Competing interests}

The authors declare that they have no competing interests.

\section{Authors' contributions}

LF contributed to the concepts of the study. $\sqcup$ performed the experiments and data collection. The experiments were designed by LF and MJ. MJ and $L J$ also participated in data analysis and interpretation. MX was responsible for EMG experiment. GDY and LGM contributed to immunohistochemistry and image processing. TXD was responsible for the pharmacological aspects of the experiments. WY contributed to data evaluation, manuscript preparation, editing and review. All authors read and approved the final manuscript.

\section{Acknowledgements}

We are grateful for the technical support provided by Dr. Feng-Yun Wang and Dr. Nan Kang (Xiyuan Hospital, affiliated with Chinese Academy of TCM, Beijing, China) and Xiang-zhu Yang (Beijing University of Chinese Medicine, Beijing, China).

This research was supported by the National Basic Research Program of China (973 Program, 2013CB531703).

\section{Author details}

${ }^{1}$ School of Preclinical Medicine, Beijing University of Chinese Medicine, Beijing, China. ${ }^{2}$ Digestive Department, Xiyuan Hospital, Affiliated with Chinese Academy of TCM, Beijing, China.

Received: 19 January 2015 Accepted: 13 October 2015

Published online: 27 October 2015 


\section{References}

1. Drossman DA, Li Z, Andruzzi E, Temple RD, Talley NJ, Thompson WG, et al. U.S. householder survey of functional gastrointestinal disorders. Prevalence, sociodemography, and health impact. Dig Dis Sci. 1993;38(9):1569-80.

2. Ping L. Investigation of functional gastrointestinal disease. Pract Med. 2003:19(4):424-6

3. Xia J. Investigate the mobidity and the related factors of functional gastrointestinal disorders in officers and soldiers at grass-roots units of some troops. J Nav Gen Hosp. 2010;23(4):193-5.

4. Zhang M. Investigation of functional gastrointestinal disease in crew. Naut Med Hyperb Med. 2011;18(1):27-30.

5. Tack J, Bisschops R, Sarnelli G. Pathophysiology and treatment of functional dyspepsia. Gastroenterology. 2004;127(4):1239-55.

6. Futagami S, Shimpuku M, Yin Y, Shindo T, Kodaka Y, Nagoya H, et al. Pathophysiology of functional dyspepsia. J Nippon Med Sch. 2011;78(5):280-5.

7. Timmermans JP, Scheuermann DW, Stach W, Adriaensen D, De Groodt-Lasseel $\mathrm{MH}$. Functional morphology of the enteric nervous system with special reference to large mammals. Eur J Morphol. 1992;30(2):113-22.

8. Costa M, Brookes SJ, Hennig GW. Anatomy and physiology of the enteric nervous system. Gut. 2000;Suppl 4:iv15-9.

9. Furness JB. The organisation of the autonomic nervous system: peripheral connections. Auton Neurosci. 2006;130(1-2):1-5.

10. Konturek SJ, Konturek JW, Pawlik T, Brzozowski T. Brain-gut axis and its role in the control of food intake. J Physiol Pharmacol. 2004;55(1):137-54.

11. Mazur M, Furgała A, Thor PJ. Visceral sensitivity disturbances in the pathogenesis of functional gastrointestinal disorders. Folia Med Cracov. 2004;45(1):33-49.

12. Holzer P, Farzi A. Neuropeptides and the microbiota-gut-brain axis. Adv Exp Med Biol. 2014;817:195-219.

13. Kojima M, Hosoda H, Date Y, Nakazato M, Matsuo H, Kangawa K. Ghrelin is a growth-hormone-releasing acylated peptide from stomach. Nature. 1999:402(6762):656-60.

14. Rindi G, Necchi V, Savio A, Torsello A, Zoli M, Locatelli V, et al. Characterisation of gastric ghrelin cells inman and other mammals: studies in adult and fetal tissues. Histochem Cell Biol. 2002;117(6):511-9.

15. Koleva DI, Orbetzova MM, Atanassova PK. Adipose tissue hormones and appetite and body weight regulators in insulin resistance. Folia Med. 2013;55(1):25-32.

16. Atalayer D, Gibson C, Konopacka A, Geliebter A. Ghrelin and eating disorders. Prog Neuropsychopharmacol Biol Psychiatry. 2013:40:70-82.

17. Komarowska H, Jaskula M, Stangierski A, Wasko R, Sowinski J, Ruchala M. Influence of ghrelin on energy balance and endocrine physiology. Neuro Endocrinol Lett. 2012;33(8):749-56.

18. Akamizu T, Iwakura H, Ariyasu H, Kangawa K. Ghrelin and functional dyspepsia. Int J Pept. 2010;2010:548457.

19. Dockray GJ. Cholecystokinin and gut-brain signalling. Regul Pept. 2009;155(1-3):6-10.

20. Chandra R. Current opinion in endocrinology. Diabetes Obes. 2007:14(1):63-7.

21. Gibbs J, Young RC, Smith GP. Cholecystokinin decreases food intake in rats J Comp Physiol Psychol. 1973;84(3):488-95.

22. Degen $L$, Matzinger D, Drewe J, Beglinger C. The effect of cholecystokinin incontrolling appetite and food intake in humans. Peptides. 2001;22(8):1265-9.

23. Khoo J, Rayner CK, Feinle-Bisset C. Gastrointestinal hormonal dysfunction in gastroparesis and functional dyspepsia. Neurogastroenterol Motil. 2010;22(2):1270-8.

24. Dockray GJ. Luminal sensing in the gut: an overview. J Physiol Pharmacol. 2003;54 Suppl 4:9-17.

25. Tomita R. Regulation of vasoactive intestinal peptide and substance $P$ in the human pyloric sphincter. Hepatogastroenterology. 2009:56(94-95):1403-6.

26. Gańko M, Całka J. Prolonged acetylsalicylic-acid-supplementation-induced gastritis affects the chemical coding of the stomach innervating vagal efferent neurons in the porcine dorsal motor vagal nucleus (DMX). J Mol Neurosci. 2014:54(2):188-98.

27. Xiao Y, Liu YY, Yu KQ, Ouyang MZ, Luo R, Zhao XS. Chinese herbal medicine liu jun zi tang and xiang sha liu jun zi tang for functional dyspepsia: metaanalysis of randomized controlled trials. Evid Based Complement Alternat Med. 2012;2012:936459.

28. Liu LS, Winston JH, Shenoy MM, Song GQ, Chen JD, Pasricha PJ. A rat model of chronic gastric sensorimotor dysfunction resulting from transient neonatal gastric irritation. Gastroenterology. 2008;134(7):2070-9.
29. Towell A, Muscat R, Willner P. Effects of pimozide on sucrose consumption and preference. Psychopharmacology (Berl). 1987;92(2):262-4

30. Strekalova T, Spanagel R, Bartsch D, Henn FA, Gass P. Stress-induced anhedonia in mice is associated with deficits in forced swimming and exploration. Neuropsychopharmacology. 2004;29(11):2007-17.

31. Wang X, Zhao T, Qiu Y, Su M, Jiang T, Zhou M, et al. Metabonomics approach to understanding acute and chronic stress in rat models. J Proteome Res. 2009:8(5):2511-8

32. Winston JH, Sarna SK. Developmental origins of functional dyspepsia-like gastric hypersensitivity in rats. Gastroenterology. 2013;144(3):570-9.

33. Halland M, Almazar A, Lee R, Atkinson E, Larson J, Talley NJ, et al. A case-contro study of childhood trauma in the development of irritable bowel syndrome. Neurogastroenterol Motil. 2014;26(7):990-8.

34. Martín-Villa JM. Neuroendocrine stimulation of mucosal immune cells in inflammatory bowel disease. Curr Pharm Des. 2014;20(29):4766-73.

35. Peruzzo B, Pastor FE, Blázquez JL, Schöbitz K, Peláez B, Amat P, et al. A second look at the barriers of the medial basal hypothalamus. Exp Brain Res. 2000;132(1):10-26.

36. Murphy KG, Dhillo WS, Bloom SR. Gut peptides in the regulation of food intake and energy homeostasis. Endocr Rev. 2006;27(7):719-27.

37. Liu L, Li Q, Sapolsky R, Liao M, Mehta K, Bhargava A, et al. Transient gastric irritation in the neonatal rats leads to changes in hypothalamic CRF expression, depression-and anxiety-like behavior as adults. PLoS One. 2011;6(5):e19498.

38. Tack J, Caenepeel P, Fischler B, Piessevaux H, Janssens J. Symptoms associated with hypersensitivity to gastric distention in functional dyspepsia. Gastroenterology. 2001;121(3):526-35.

39. Vermeulen W, De Man JG, Pelckmans PA, De Winter BY. Neuroanatomy of lower gastrointestinal pain disorders. World J Gastroenterol. 2014;20(4):1005-20.

40. Vandenberghe J, Vos R, Persoons P, Demyttenaere K, Janssens J, Tack J. Dyspeptic patients with visceral hypersensitivity: sensitisation of pain specific or multimodal pathways? Gut. 2005;54(7):914-9.

41. Liu LS, Shenoy M, Pasricha PJ. The analgesic effects of the GABAB receptor agonist, baclofen, in a rodent model of functional dyspepsia. Neurogastroenterol Motil. 2011;23(4):356-61.

42. Tack J, Talley NJ, Camilleri M, Holtmann G, Hu P, Malagelada JR, et al. Functional gastroduodenal disorders. Gastroenterology. 2006;130(5):1466-79.

\section{Submit your next manuscript to BioMed Central and take full advantage of:}

- Convenient online submission

- Thorough peer review

- No space constraints or color figure charges

- Immediate publication on acceptance

- Inclusion in PubMed, CAS, Scopus and Google Scholar

- Research which is freely available for redistribution 